\title{
Panorama de la Innovación Docente en la Universidad Española. Perspectivas desde la Universidad de Cádiz ${ }^{1}$
}

Panorama of Teaching Innovation at the Spanish University. Perspectives from the University of Cadiz

\author{
Juan Antonio Poce Fatou ${ }^{2}$ \\ Universidad de Cádiz \\ juanantonio.poce@uca.es
}

\section{Resumen}

En este trabajo se identifican los orígenes de la Innovación Docente en el sistema universitario español y su concreción en la Universidad de Cádiz. Se describe el panorama actual de la Innovación Docente en ambos contextos, acompañándolo de datos de evolución del programa desarrollado en la Universidad de Cádiz desde el curso 2011/12 hasta el 2018/19. Se reflexiona sobre el alcance del concepto Innovación Docente y sobre los objetivos que las universidades del Espacio Europeo de Educación Superior deberán afrontar en los próximos años.

Palabras clave: Declaración de Bolonia, Educación Superior, EEES, Innovación Docente, Universidad de Cádiz

\begin{abstract}
This work identifies the origins of Teaching Innovation in the Spanish university system and its concretion in the University of Cadiz. It describes the current panorama of Teaching Innovation in both contexts, accompanied by data on the evolution of the program developed at the University of Cadiz from the 2011/12 to the 2018/19 academic year. It reflects on the

${ }^{1}$ Recibido: 29/11/2019 Evaluado: 15/01/2020 Aceptado: 21/02/2020

${ }^{2}$ Director de la Unidad de Innovación Docente de la Universidad de Cádiz desde junio de 2011 hasta abril de 2015 y Vicerrector de Recursos Docentes y de la Comunicación desde entonces hasta julio de 2019.
\end{abstract}


scope of the concept of Teaching Innovation and on the objectives that the universities of the European Higher Education Area will have to face in the coming years.

Keywords: Bologna Declaration, EHEA, Higher Education, Teaching Innovation, University of Cadiz

\section{El Espacio Europeo de Educación Superior}

En mayo de 1988 los ministros encargados de la educación superior en Alemania, Francia, Italia y Reino Unido suscribieron en París la conocida como Declaración de la Sorbona (Allegre, Berlinguer, Blackstone y Rüttgers, 1998), instando al desarrollo de un Espacio Europeo de Educación Superior (EEES). Un año más tarde, los ministros de 29 países europeos celebraron una conferencia en Bolonia (Einem et al., 1999) que sentó las bases para la construcción definitiva del EEES con fecha límite de 2010.

Si bien en los últimos años del siglo XX comenzaban a vislumbrarse cambios en la docencia universitaria, siempre al hilo de aquel mantra doble que representaba la Declaración de Bolonia y el año 2010, hasta 2007 no se publicó el Real Decreto 1393/2007, de 29 de octubre, por el que se establecía la ordenación de las enseñanzas universitarias oficiales (Cabrera, 2007), un documento que constituye un marco normativo que concreta en una serie de tareas específicas el objetivo general de crear un Espacio Europeo de Educación Superior.

El Real Decreto establecía objetivos de gran calado basados en el concepto de Crédito Europeo, unidad de medida del haber académico que representa la cantidad de trabajo del estudiante para cumplir los objetivos del programa de estudios, definida años antes en el Real Decreto 1125/2003, de 5 de septiembre, por el que se establecía el sistema europeo de créditos y el sistema de calificaciones en las titulaciones universitarias de carácter oficial con validez en todo el territorio nacional (Castillo, 2003). Entre aquellos objetivos cabe mencionar dos bloques de cambios estructurales:

a. Los que afectarían a los estudios de grado, máster y doctorado para facilitar la coordinación entre las universidades del Espacio Europeo de Educación Superior.

b. Los que afectarían a la creación de los sistemas de Garantía de la Calidad como parte de los nuevos planes de estudio, para que la nueva organización de las enseñanzas pudiese funcionar eficientemente y crear la confianza necesaria sobre el proceso de acreditación de títulos.

\section{Orígenes de la Innovación Educativa en la Educación Superior}

No obstante, no todos los cambios que se avecinaban eran estructurales. El Real Decreto 1393/2007 también impulsaba un cambio en las metodologías docentes centrado en el aprendizaje del estudiante a lo largo de la vida. Para ello, los títulos deberían tener en el centro de sus objetivos la adquisición de competencias ampliando, sin excluir, el tradicional enfoque basado en contenidos y horas lectivas, pero haciendo énfasis en los métodos de 
aprendizaje de dichas competencias, así como en los procedimientos para evaluar su adquisición.

Quizá sea este contenido de la normativa el que podría ser considerado como referencia sobre la que se construyeron los programas de Innovación Docente que las universidades públicas del estado español ya empezaban a vislumbrar desde años anteriores a 2007 gracias a fondos presupuestarios de la Unión Europea, el Estado, la Administración autonómica y la propia Universidad.

Aquellos fondos y el interés por cimentar el Espacio Europeo se concretaron en la Universidad de Cádiz en un programa denominado Proyecto Europa que en 2005 publica la primera convocatoria para el desarrollo de proyectos de Innovación Docente entre el profesorado y gestiona cuatro más hasta el curso 2010/11.

En 2011 la oficina del Proyecto Europa se transforma en la Unidad de Innovación Docente que hoy en día es parte estructural de la Universidad de Cádiz asumiendo el soporte técnico en las iniciativas del equipo de gobierno para el fomento de la innovación docente y los programas de formación del profesorado.

Desde 2005 en la Universidad de Cádiz se han publicado catorce bases de convocatorias para incentivar la innovación docente. Se trata de un periodo suficientemente amplio para crear perspectiva y propiciar el análisis de la Innovación Docente que se expone en estas líneas.

\section{El eufemismo de la Innovación Docente}

La preocupación por la docencia siempre ha estado presente en mayor o menor grado entre los profesionales de la enseñanza, pero no fue hasta el desarrollo de la iniciativa de creación de un Espacio Superior, tras la Declaración de Bolonia, que esta preocupación se generaliza y alcanza niveles de interés sin precedentes. Es entonces cuando se cae en la cuenta de que, en buena parte de la Universidad Española, hasta aquellos momentos la preocupación por la enseñanza no iba mucho más allá de asegurar que se impartieran las clases.

La enseñanza constituye una tarea compleja que establece un objetivo fundamental y prioritario: el aprendizaje del estudiante. Se trata de una tarea ardua en cualquier nivel educativo y no lo es menos en el ámbito de la Educación Superior. El éxito o el fracaso se ven influidos por innumerables factores, algunos independientes entre sí, otros dependientes en niveles difíciles de cuantificar. El espacio físico donde se imparte la docencia, la ratio profesor - estudiantes, la formación previa del estudiante, la metodología docente, la interacción a través de tutorías u otros medios de comunicación, el uso de las TIC, la coordinación de los temarios con otras asignaturas relacionadas, el desarrollo de prácticas, o la propia rama de conocimiento en la que se enmarca la asignatura pueden influir significativamente en la consecución del éxito o del fracaso y ser parámetros susceptibles de estudio en un proyecto de Innovación Docente. 
Más allá de mantener una actividad inercial de trabajo carente de mecanismos para medir su eficacia, los nuevos programas de innovación estimulan al profesorado a realizar autocrítica, a reflexionar sobre las posibilidades de modificar la metodología o el sistema de evaluación, o a incluir elementos que hasta el momento no solían entrar en la fórmula: la motivación, la coordinación con otras asignaturas, la conexión del temario y de las actividades con el mundo exterior. Por esa razón cabe plantearse si esos programas de Innovación Docente motivados por la Declaración de Bolonia representan en sí mismos un eufemismo que trata de disfrazar, bajo el concepto innovación, lo que en realidad constituía un toque de atención de las instancias europeas para que las instituciones de Educación Superior dedicaran más esfuerzos y recursos a la actividad docente.

\section{Los límites de la Innovación Docente}

Y en esta línea de pensamiento merece la pena recapacitar sobre el recorrido que el concepto innovación tiene en la enseñanza superior.

La alteración de algo en la docencia, que introduzca novedades, no es en sí mismo el objetivo a alcanzar. Innovación sí, pero solo si representa una mejora en las perspectivas de aprendizaje del estudiante y, es más, si la innovación es fructífera, mejor consolidarla que volver a propiciar cambios que puedan restar valor a lo ya conseguido.

Por esta razón la palabra mejora está implícita en los programas de innovación docente desarrollados en la Universidad de Cádiz en las últimas nueve convocatorias. La mejora es, en consecuencia, el fin último, no la innovación.

Siendo este el objetivo principal, fomentar el cambio se ha llevado a cabo en prácticamente el $100 \%$ de las universidades públicas españolas a base de programas de Innovación Docente apoyados en fondos presupuestarios que no fueron escasos (en el periodo 2005-2010), aunque algunas universidades pudiesen haberlo dedicado a otros menesteres diferentes a los originalmente definidos por los organismos financiadores.

\section{La experiencia en la Universidad de Cádiz (2011-2019)}

Ahondando en lo anterior, si la financiación no ha constituido en términos generales un problema para dotar programas con los que incentivar en el profesorado la mejora docente, el reto en los últimos años se concretó en dar con los elementos que estimulasen la participación. La política desarrollada con esta finalidad en la Universidad de Cádiz desde el curso 2011/12 se concretó en convocatorias (Unidad de Innovación Docente, 2011) publicadas anualmente hasta el curso 2019/20 construidas sobre los siguientes pilares:

- Un programa formativo para aportar ideas y conocimientos al profesorado en sus facetas de gestión, investigación y docencia que ha apostado por las nuevas tendencias en innovación docente con una oferta ligada a las nuevas tecnologías, pluringüismo, coaching educativo, clase invertida, gamificación, etc.

- Convocatorias competitivas para solicitar proyectos que propicien:

o La innovación o la mejora de la metodología docente (convocatoria Innova). 
o La innovación o la mejora de otros aspectos de la docencia no vinculados a la metodología como por ejemplo la creación de materiales, herramientas de evaluación, sistemas de coordinación entre asignaturas, entre grados, ... (convocatoria Actúa).

o La puesta en funcionamiento de proyectos formativos solicitados por los propios profesores, que por su especificidad o adecuación a áreas de conocimiento concretas, no tienen cobertura en el programa formativo general (convocatoria Fórmate).

o La creación de productos audiovisuales destinados a cursos MOOC (Convocatoria MOOC).

- Herramientas para facilitar la difusión de los resultados:

o Convocatoria Difunde.

o Publicación en abierto de las memorias de proyectos ejecutados en la web de la Unidad de Innovación Docente (Unidad de Innovación Docente, 2012).

o Jornadas de Innovación Docente (Jornadas de Innovación Docente, 2016).

- Incentivos para implicar a los estudiantes en Innovación Docente a través de la convocatoria de premios a las mejores cápsulas audiovisuales de aprendizaje (convocatoria Cápsula).

- Un sistema de evaluación de las solicitudes y de las memorias de los proyectos (Innova, Actúa, Fórmate, Cápsula) eficaz, justo, transparente y sostenible en el tiempo.

- Un sistema de revisión y publicación periódica de las convocatorias.

- Un sistema de reconocimiento al profesorado implicado en los proyectos mejor valorados por la comisión competente basado en la comunicación a toda la comunidad universitaria de la relación de proyectos y profesores premiados y en la entrega de premios en acto público.

La evolución de los parámetros relacionados con estos pilares del programa arroja luz sobre la situación actual de la Innovación Docente en la Universidad de Cádiz. Se indicaba que el programa debía consolidarse sobre una oferta formativa adecuada y en este sentido cabe reflejar que la Unidad de Innovación Docente, desde el curso 2011/12 hasta el 2018/19, desplegó una oferta de $97 \pm 25$ cursos de media anual, cubriendo $1.642 \pm 319$ plazas por año (Figura 1) lo que representa a un 93,63 \% \pm 20,20 de la plantilla. Es una estimación de referencia, ya que se suma la participación de un mismo profesor en todos los cursos que ha finalizado. 


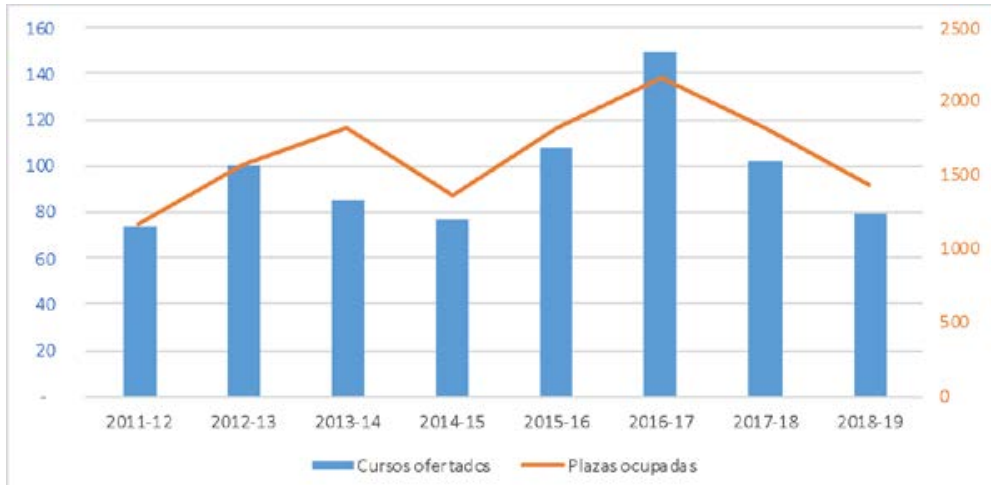

Figura 1. Cursos ofertados y plazas cubiertas en el programa de formación del profesorado de la Universidad de Cádiz entre los cursos 2011/12 y 2018/19.

Respecto a la calidad de los cursos y su adecuación a la actividad del profesorado, durante el mismo periodo la calificación media en las encuestas de satisfacción en una escala de 0 a 10 se situó en 8,91 \pm 0,13 puntos. En cuanto a la participación en proyectos de Innovación Docente, la Figura 2 muestra los datos de participación en las solicitudes aprobadas en las convocatorias Innova y Actúa.

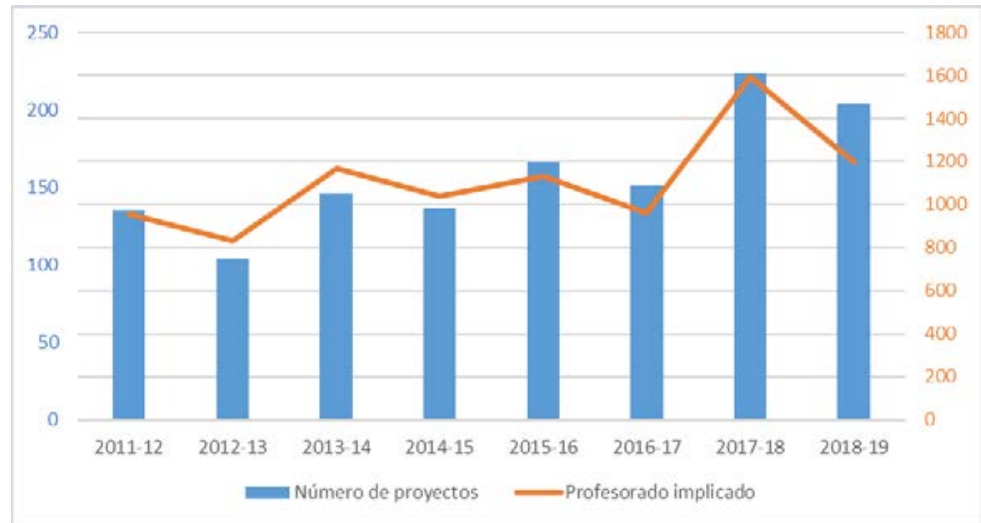

Figura 2. Proyectos aprobados en las convocatorias del programa de Innovación Docente y plazas cubiertas Leyenda: Los datos incorporan tantas veces a un profesor como en proyectos participa. 
Estos datos señalan que el soporte formativo parece haber dado cobertura a las necesidades del profesorado propiciando una implicación promedio en proyectos de Innovación Docente del 62,79 \pm 11,63\% de la plantilla, una participación que, según se desprende de la Figura 3, parece haber influido significativamente en la valoración que otorgan los estudiantes a la docencia recibida desde el curso 2011/12.

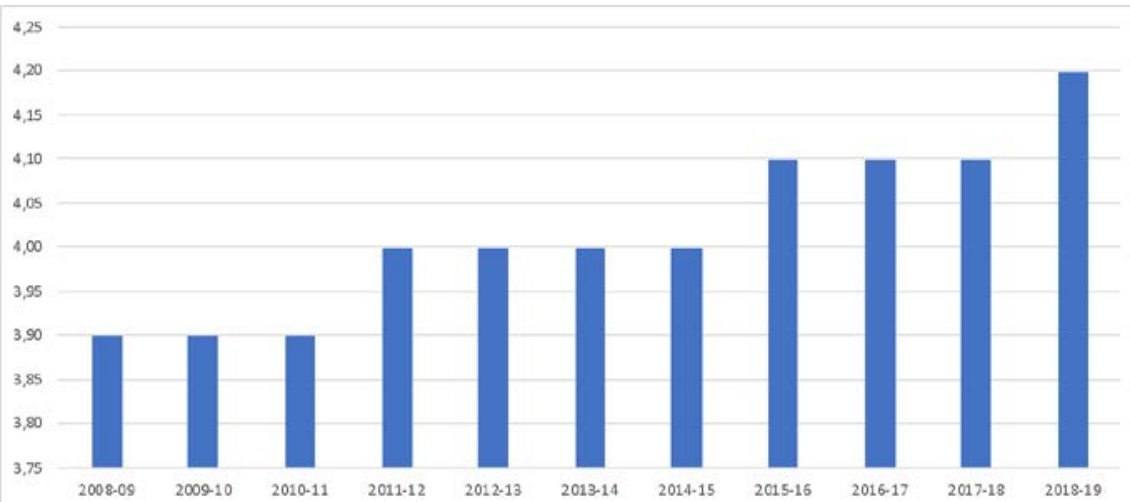

Figura 3. Valoración que otorga el alumnado de la Universidad de Cádiz al desarrollo de la docencia (escala 1-5) (datos procedentes del informe de satisfacción con la docencia universitaria)

Leyenda: Se trata de una estimación de referencia, ya que se suma la participación de un mismo profesor en todos los proyectos aceptados en los que figure.

Pero más allá de estos u otros datos que pudieran dar fe de la eficacia del programa, el elemento más importante a destacar corresponde con el sistema ideado para planificar las convocatorias y evaluar las solicitudes de manera que el incremento de carga anual fuese sostenible y no supusiese merma de su eficacia. Desde el curso 2011/12 la evaluación de las solicitudes dejó de llevarse a cabo a través de un sistema doble ciego con profesores de universidades públicas andaluzas asociados a la DEVA (Dirección de Evaluación y Acreditación. Agencia Andaluza del Conocimiento) y pasó a ser tarea de la Comisión (interna) de Innovación y Mejora Docente de la Universidad de Cádiz en la que participan miembros del equipo de gobierno con competencias en Innovación Docente, representantes de centros, directores de departamento, profesores premiados en anteriores convocatorias y personal de la Unidad de Calidad.

A partir de entonces cada solicitud se evaluó (con base en un sistema triple ciego y rúbrica consensuada) por parte de miembros de la comisión que llevaban sus consideraciones y planteamientos sobre la bondad del proyecto a una reunión en la que, partiendo de la calificación inicial otorgada, se valoraban entre todos los comisionados otros aspectos que pudiesen modificarla. 
Los argumentos esgrimidos en estas reuniones se convirtieron en elemento esencial para dotar de calidad la resolución final y para el óptimo aprovechamiento de los recursos disponibles en la universidad. La amplia variedad de peticiones y proyectos planteaba en no pocos casos, necesidades o herramientas que ya estaban disponibles en la institución pero que no eran conocidas por el solicitante. El amplio espectro de conocimientos y gestión abarcado por los miembros PAS y PDI de la comisión facilitaba la búsqueda de soluciones y puede ser considerada como argumento de peso para entender por qué la política puesta en funcionamiento en el curso 2011/12 sigue vigente en el 2019/2020.

La misión de esta comisión no solo se centró en la evaluación de las solicitudes, también evaluó las memorias (para clasificarlas por niveles de calidad y retirar el reconocimiento a aquellas que no alcanzaron el nivel mínimo) y retocó año tras año el texto de las convocatorias para adaptarlas a los tiempos y necesidades planteadas por profesorado y estudiantes.

Respecto al poder de convocatoria y a la vista de los datos de la Figura 2 y de la Figura 3, cabe pensar que la política puesta en marcha en la Universidad de Cádiz alcanzó los objetivos inicialmente planteados.

\section{Lo que la Innovación Docente no abarca}

Con base en el Real Decreto 1393/2007 las universidades públicas españolas recibieron fondos para incentivar la Innovación Docente que se concretaron mayoritariamente en políticas basadas en la voluntariedad del profesorado por afrontar proyectos de mejora.

Esas políticas en la Universidad de Cádiz han alentado la participación del profesorado, pero también la difusión de los resultados y la interacción a través de las Jornadas de Innovación Docente celebradas en los años 2016, 2017 y 2018. En ambos casos, al difundir en abierto tanto el contenido de las memorias de proyectos como de las jornadas, la comunidad universitaria y la sociedad en general tiene acceso a toda la variedad de retos y contextos docentes que han protagonizado los proyectos llevados a cabo.

El profesorado que participa en las convocatorias de Innovación Docente o en estas jornadas se caracteriza por estar especialmente motivado y preocupado por su actividad. Sus escenarios de trabajo y los retos que afrontan son públicos. Por sus memorias pueden conocerse sus estrategias docentes, sus experiencias y la valoración que les dieron los alumnos implicados, pero, ¿qué pasa con aquellos profesores, con aquellas asignaturas que no participan en convocatorias de Innovación Docente y quedan fuera de los circuitos voluntarios de mejora?

Aún sin disponer de datos de primera mano, pero sí multitud de indicios y evidencias, cabe pensar que la mayor parte son profesores que no presentan ningún tipo de incidencia reseñable en su actividad docente. Constituyen un amplio espectro que abarca entre profesores de calidad media y profesores excelentes que no encuentran incentivo particular en participar en proyectos de innovación (lo que no quiere decir que no trabajen en sus propios proyectos personales al margen de las convocatorias oficiales). 
No obstante, un pequeño número de profesores sí que genera distorsiones según se refleja en las memorias anuales de la Inspección General de Servicios. Distorsiones relacionadas con desviaciones significativas entre los contenidos de las asignaturas y los resultados del aprendizaje o con la aplicación de los sistemas de evaluación, que pueden llevar, por ejemplo, a que dos grupos de una misma asignatura impartidos por sendos profesores en el mismo curso académico alcancen cifras diametralmente opuestas en las tasas de rendimiento.

\section{El camino hacia la excelencia}

Si bajo el concepto de Innovación Docente nos contentamos con incentivar la participación y el desarrollo en nuevos proyectos, en línea con lo que establece el documento Criterios y directrices para el aseguramiento de la calidad en el Espacio Europeo de Educación Superior de 2015, la Universidad de Cádiz está cubierta, ya que el documento establece con respecto al personal docente la necesidad de "Fomentar la actividad intelectual para reforzar el vínculo entre educación e investigación”, así como "la innovación en los métodos de enseñanza y el uso de nuevas tecnologías” (ENQA, 2015, p.17).

Cuestiones estas ampliamente incentivadas en los últimos años. Pero si nos marcamos el reto de que la Innovación Docente se constituya en un mecanismo esencial para optimizar la calidad de las enseñanzas y el aprendizaje de nuestros alumnos, quizá deberíamos avanzar aún más acudiendo a lo que, sobre el personal docente, indicaba la versión de 2005 de este mismo informe:

Para muchos estudiantes, los profesores son el recurso de aprendizaje más importante. Es fundamental que los docentes tengan un conocimiento y comprensión completos de la materia que enseñan, que dispongan de las habilidades y experiencia para transmitir sus conocimientos, comprendan eficazmente a los estudiantes en una variedad de contextos de aprendizaje y que puedan obtener retroalimentación sobre su propia actuación ... Deben darse oportunidades al personal docente para que desarrolle y amplíe su capacidad de enseñanza y estimularles para que saquen partido de sus habilidades. Las instituciones deben proporcionar oportunidades a los profesores de bajo rendimiento para que mejoren sus habilidades de forma que alcancen un nivel aceptable. Deben, así mismo, disponer de los medios que permitan darles de baja de sus funciones docentes si se demuestra que continúan siendo ineficaces (ENQA, 2005, p. 18).

La tarea no es sencilla y quizá sea esa la razón de que esta redacción haya desaparecido en la versión de 2015. Las normativas estatales que dan cobertura al funcionariado hacen prácticamente imposible dar de baja de sus funciones docentes a profesores ineficaces pero quizá deberíamos tomar nota para que desde los servicios de inspección de las universidades, en colaboración con los Sistemas de Garantía de Calidad, se dispusiesen los mecanismos necesarios para identificar problemas asociados a la capacidad del profesorado y darles solución dentro del marco normativo vigente con la mayor transparencia y justicia para el sistema público de enseñanza (partiendo por ejemplo de una nueva encuesta diseñada por profesionales para conocer el nivel de satisfacción de los estudiantes con la docencia recibida y de la ampliación de los servicios de inspección para disponer de asesoramiento pedagógico). Esta circunstancia estaría amparada por el propio estatuto de la Universidad de 
Cádiz que establece para su profesorado el deber de someterse a los procedimientos y sistemas de evaluación de su rendimiento que se establezcan por el Consejo de Gobierno (Universidad de Cádiz, 2017, p. 41)

\section{La profesión docente}

El profesor universitario afronta tareas docentes, de investigación y en no pocas ocasiones también de gestión para dar sentido a la autonomía de la que goza la Universidad según lo dispuesto en la Ley Orgánica de Universidades.

La investigación constituye, por tanto, una actividad inherente al profesor que amplía significativamente su valor profesional. Cuando se afronta una investigación, los primeros pasos se basan en la observación del fenómeno analizado y en la búsqueda de información que arroje luz sobre el problema a desentrañar. La búsqueda de soluciones pasa por el diseño de hipótesis, el planteamiento de objetivos y en gran parte, por la experimentación. Por esto el investigador está continuamente actualizándose, recabando las últimas publicaciones del momento para visualizar el avance del conocimiento en una temática determinada. Lamentablemente, esa actualización constante no siempre contagia la propia labor docente de ese mismo investigador que a veces se desarrolla sobre temarios desfasados o metodologías docentes insuficientemente adaptadas a los tiempos, una circunstancia que los programas de Innovación Docente han ayudado a cambiar.

Cada seis años el investigador expone parte de su trabajo a valoración de la Comisión Nacional Evaluadora de la Actividad Investigadora (CNEAI) sometiéndose a unos criterios muy exigentes. Cada cinco años la misma persona en su vertiente de profesor expone su actividad docente en su propia universidad sin que concurra (salvo en pocas instituciones del territorio nacional) limitación alguna para que se le otorgue valoración positiva de forma prácticamente automática. Sexenios y quinquenios concurren entonces en una misma persona con gestiones, administraciones, exigencias y respuestas totalmente diferentes que dotan a la Investigación del caché e importancia del que, entre todos, privamos a la enseñanza.

El perfil investigador es clave en la trayectoria del profesor universitario. Es lo que lo distingue del profesor de bachillerato o de Enseñanza Secundaria, de Formación Profesional o de Escuela Oficial de Idiomas y lo que le hace estar siempre situado en la vanguardia del conocimiento. El Real Decreto-ley 14/2012, de 20 de abril, de medidas urgentes de racionalización del gasto público en el ámbito educativo (Rajoy, 2012) ligó la capacidad docente al resultado de la evaluación de los sexenios de investigación. Bajo esta consideración, aquellos profesores que no cuentan con un sexenio vivo tienen que impartir más docencia que aquellos que sí lo acreditan. En consecuencia, como incentivo, a los “mejores investigadores” se les reduce la “carga” docente en una proporción que es asumida por aquellos menos involucrados con la investigación.

Destinamos entonces a la docencia y a la formación de los estudiantes al profesorado que, según los criterios de CNEAI, está menos involucrado, menos actualizado, menos ligado a ese dinamismo intelectual que proporciona la investigación. Teniendo en cuenta, en cualquier caso, que la falta de producción investigadora no implica que el profesorado no esté actualizado en los contenidos y materias que imparte, la aplicación del Real Decreto-ley 
14/2012 con base en méritos investigadores, ¿no va en detrimento del servicio que presta la Universidad a través de la docencia?

En los últimos años el concepto “carga” docente está haciendo un flaco favor al sistema universitario español. Las distintas versiones de Planes de Dedicación Académica del PDI valoran las actividades que éste realiza para traducirlas en descuento de la actividad docente. Un descuento que se reclama por regla general desde todos los sectores del profesorado, sin darnos cuenta que el profesor universitario es, ante todo, profesor, y que la enseñanza no es una carga a soportar sino un privilegio del que disfrutar.

Quizá la próxima reforma universitaria debiera considerar volver a establecer una misma carga docente para todo el profesorado, eso sí, elevando sustancialmente el celo que actualmente se aplica a la evaluación de los méritos docentes.

\section{Conclusiones}

Bajo el concepto de Innovación Educativa los países impulsores del Espacio Europeo de Educación Superior lograron que la enseñanza cobrase protagonismo en la Universidad y se crearan programas dirigidos al profesorado para impulsar la renovación metodológica y la mejora continua.

La innovación en la docencia constituye un medio para consolidar la mejora y las buenas prácticas. Estos, y no la introducción continua de novedades en la docencia, son en última instancia los objetivos que persiguen los programas de Innovación Docente. Allá donde se logre la maximización del aprendizaje y la adquisición de las competencias previstas en una asignatura, es recomendable no afrontar más cambios sino consolidar los ejecutados y difundir los resultados de la experiencia para beneficio de otros docentes y estudiantes.

Por otra parte, mediante convocatorias que potencien la innovación en la docencia es difícil localizar problemas existentes en la docencia de asignaturas concretas, simplemente porque su profesorado no suele participar en este tipo de iniciativas. Para localizar estas situaciones y atenderlas debidamente se requiere la coordinación entre los correspondientes Sistemas de Garantía de Calidad y los servicios de inspección de las instituciones.

Los programas de Innovación Docente desplegados en las Universidades Españolas ni han sido diseñados desde las mismas perspectivas políticas ni con las mismas intensidades y recursos, es por ello que es difícil cuantificar el beneficio global que han supuesto para el conjunto del sistema universitario. En la Universidad de Cádiz se ha constatado que la participación del profesorado en los programas de Innovación Docente no depende tanto de la financiación disponible como de la existencia de políticas estimulantes (en la convocatoria Actúa del curso 2019/20 el 33,33\% de las solicitudes no requerían financiación mientras que en la convocatoria Innova el porcentaje se extiende hasta el 75,43\%) apoyadas en el trabajo de una comisión evaluadora de carácter interno constituida por miembros del profesorado y del personal de administración y servicios.

Es difícil medir objetivamente el beneficio que los programas de Innovación Docente han tenido en la docencia. Se trata de un reto a afrontar que requiere un esfuerzo considerable en 
personal y recursos. Para realizar esta estimación es importante dar peso a la opinión de los estudiantes y en este sentido, en la Universidad de Cádiz, cabe mencionar que el nivel de satisfacción de los estudiantes con la docencia ha ido incrementándose desde la puesta en marcha del actual programa de Innovación Docente en el curso 2011/12, lo que representa un claro indicio de su eficacia.

En cuanto a los retos más inmediatos que la Innovación Docente debería afrontar en los próximos años se encuentra el de asegurar mediante las políticas adecuadas que no cesen las inquietudes del profesorado por mejorar en su labor docente y el de no perjudicar el prestigio de la enseñanza universitaria por la aplicación de criterios exclusivamente centrados en la labor investigadora.

\section{Referencias}

Allegre, C., Berlinguer, L., Blackstone, T. and Rüttgers, J. (1998). Sorbonne Joint Declaration. Joint declaration on harmonization of the architecture of the European higher education system. Paris: The European Higher Education Area. Recuperado de https://cutt.ly/je1NEpe, consultado el 18/04/2020.

Cabrera, M. (2007). Real Decreto 1393/2007, de 29 de octubre, por el que se establece la ordenación de las enseñanzas universitarias oficiales. Boletín Oficial del Estado. Recuperado de https://cutt.ly/7e1NUfm, consultado el 18/4/2020.

Castillo, P. (2003). Real Decreto 1125/2003, de 5 de septiembre, por el que se establece el sistema europeo de créditos y el sistema de calificaciones en las titulaciones universitarias de carácter oficial y validez en todo el territorio nacional. Boletín Oficial del Estado. Recuperado de https://cutt.ly/Ze1NSyu, consultado el 18/4/2020.

Einem, C., Ade, J., Schmit, G., Zeman, E., Totomanova A. M., Lukas, T., Vestager M., Allegre C. Rask M., Erdsiek-Rave, U., Catenhusen, W., Kiss A., Arsenis, G., Dowling, P., Sigurdardottir, G., Kokek, T., Zecchino, O., Hennicot-Schoepges, E., Platelis, K., Hermans, L., Galea, L., Winkler, W., Lilletun, J., Marga, A., Grilo, E. M., Zgaga, P., Ftacnik, M., Bladh, A., Fernández Díaz, J., Blackstone, T., Kleiber, C. (1999). The Bologna Declaration of 19 June 1999. Joint declaration of the European Ministers of Education. Bolonia. The European Higher Education Area. Recuperado de https://cutt.ly/Se1NTQ6, consultado el 18/4/2020.

ENQA. European Association for Quality Assurance in Higher Education (2005). European Higher Education Area and Bologna Process. Standards and Guidelines for Quality Assurance in the European Higher Education Area. Recuperado de https://cutt.ly/Rt7SKWG, consultado el 18/4/2020. 
ENQA. European Association for Quality Assurance in Higher Education (2015). Criterios y directrices para el aseguramiento de Calidad en el Espacio Europeo de Educación Superior (ESG). Recuperado de https://cutt.ly/ke04AtJ, consultado el 18/4/2020.

Jornadas de Innovación Docente (2016). Ediciones 2016-2019. Universidad de Cádiz. Recuperado de https://cutt.ly/2t73182, consultado el 18/4/2020.

Rajoy, M. (2012). Real Decreto-ley 14/2012, de 20 de abril, de medidas urgentes de racionalización del gasto público en el ámbito educativo. Boletín Oficial del Estado. Recuperado de https://cutt.ly/ee2iPBH, consultado el 18/4/2020.

Unidad de Innovación Docente (2011). Convocatorias anteriores. Universidad de Cádiz. Recuperado de https://cutt.ly/Nt73nzo, consultado el 18/4/2020.

Unidad de Innovación Docente (2012). Buscador de Memorias de Innovación. Universidad de Cádiz. Recuperado de https://cutt.ly/mt73ETZ, consultado el 18/4/2020.

Universidad de Cádiz (2017). Estatutos de la Universidad de Cádiz. Recuperado de https://cutt.ly/Be04ZRw, consultado el 18/4/2020. 